\title{
SOME HOMOTOPY THEORETICAL QUESTIONS ARISING IN NIELSEN COINCIDENCE THEORY
}

\author{
ULRICH KOSCHORKE \\ Fachbereich Mathematik, Universität Siegen \\ 57068 Siegen, Germany \\ E-mail: koschorke@mathematik.uni-siegen.de
}

\begin{abstract}
Basic examples show that coincidence theory is intimately related to central subjects of differential topology and homotopy theory such as Kervaire invariants and divisibility properties of Whitehead products and of Hopf invariants. We recall some recent results and ask a few questions which seem to be important for a more comprehensive understanding.
\end{abstract}

Throughout this paper $M$ and $N$ will denote closed connected smooth manifolds of dimensions $m$ and $n \geq 2$, resp.

Definition 1 (cf. [K2], (2), (3) and 1.1). Given (continuous) maps $f_{1}, f_{2}: M \rightarrow N$, let $\operatorname{MC}\left(f_{1}, f_{2}\right)$ and $\operatorname{MCC}\left(f_{1}, f_{2}\right)$, resp., denote the minimum number of points and of path components, resp., among all the coincidence subspaces

$$
\mathrm{C}\left(f_{1}^{\prime}, f_{2}^{\prime}\right)=\left\{x \in M \mid f_{1}^{\prime}(x)=f_{2}^{\prime}(x)\right\} \subset M
$$

of maps $f_{i}^{\prime}$ which are homotopic to $f_{i}, i=1,2$.

The pair $\left(f_{1}, f_{2}\right)$ is called loose if $\operatorname{MC}\left(f_{1}, f_{2}\right)=0$ (or, equivalently, $\operatorname{MCC}\left(f_{1}, f_{2}\right)=0$ ), i.e. if the maps $f_{1}$ and $f_{2}$ can be deformed so as to be away from one another.

The principal aim in topological coincidence theory is to get a good understanding of the minimum numbers $\operatorname{MC}\left(f_{1}, f_{2}\right)$ and $\operatorname{MCC}\left(f_{1}, f_{2}\right)$ (compare [B], p. 9) and, in particular, to obtain precise looseness criteria.

In [K2] a geometric invariant $\omega^{\#}\left(f_{1}, f_{2}\right)$ was introduced: it is the bordism class of the (generic) coincidence submanifold

$$
C=\mathrm{C}\left(f_{1}, f_{2}\right)=\left\{x \in M \mid f_{1}(x)=f_{2}(x)\right\}
$$

2000 Mathematics Subject Classification: Primary 54H25, 55M20; Secondary 55P35, 55Q15, 55Q25, 55Q40.

Key words and phrases: coincidence invariants, Nielsen numbers, Kervaire invariants.

The paper is in final form and no version of it will be published elsewhere. 
of $M$, together with a description of its normal bundle and a map into a certain path space $E\left(f_{1}, f_{2}\right)$. Moreover, a simple numerical invariant was extracted: the Nielsen number $\mathrm{N}^{\#}\left(f_{1}, f_{2}\right)$, which counts the "essential" pathcomponents of the target space $E\left(f_{1}, f_{2}\right)$. We have

$$
\mathrm{N}^{\#}\left(f_{1}, f_{2}\right) \leq \operatorname{MCC}\left(f_{1}, f_{2}\right) \leq \operatorname{MC}\left(f_{1}, f_{2}\right) ;
$$

in many cases, e.g. in the stable range $m \leq 2 n-3$, actually $\mathrm{N}^{\#}\left(f_{1}, f_{2}\right)=\operatorname{MCC}\left(f_{1}, f_{2}\right)$ (compare [K1], 1.10).

ExAmPle 2 (Fixed point theory). Here $M=N, f_{2}=$ identity map, and $\mathrm{N}^{\#}\left(f_{1}, \mathrm{id}\right)$ is the classical Nielsen number of $f_{1}$; it is known to coincide with the minimum number of fixed points occuring in the homotopy class of $f_{1}$, provided $n \neq 2$ or $\chi(N) \geq 0$ ("Wecken theorems", cf. [B]).

In this paper we will discuss the case $M=S^{m}$ which is particularly accessible to the methods of homotopy theory. Here we can identify $\omega^{\#}\left(f_{1}, f_{2}\right)$ with the bordism class of the (generic) coincidence submanifold $\mathrm{C}\left(f_{1}, f_{2}\right)$ of $S^{m}$ together with a (nonstabilized) framing and a map into the loop space $\Omega N$ of $N$. Via the Pontryagin-Thom procedure, this can be interpreted by maps from $S^{m}$ into the Thom space of the trivial $n$-plane bundle over $\Omega N$. We obtain the homomorphism

$$
\omega^{\#}: \pi_{m}(N) \oplus \pi_{m}(N) \rightarrow \pi_{m}\left(S^{n} \wedge(\Omega N)^{+}\right)
$$

where $(\Omega N)^{+}$denotes the loop space with one point added disjointly (cf. section 6 of [K2]; for a stabilized version and its relations e.g. to Hopf-James invariants see [K1], 1.14).

From now on we consider only maps $f_{1}, f_{2}, f, \ldots: S^{m} \rightarrow N$ where we assume that the order

$$
k:=\# \pi_{1}(N)
$$

of the fundamental group of $N$ is finite (otherwise $\left(f_{1}, f_{2}\right)$ is loose for all $f_{1}, f_{1}: S^{m} \rightarrow N$, cf. [K4], 1.3). Then the exact homotopy sequence of the obvious projection $\bigvee^{k} S^{n} \vee \tilde{N}$ $\rightarrow \tilde{N}$, turned into a fibre map, yields the isomorphism

$$
\kappa: \pi_{m}\left(S^{n} \wedge(\Omega N)^{+}\right) \rightarrow \pi_{m}\left(\bigvee^{k} S^{n} \vee \tilde{N}, \tilde{N}\right)
$$

(cf. [K2], (61)) which may give useful information on the target group of $\omega^{\#}$; here $\tilde{N}$ denotes the universal covering space of $N$.

If $N$ happens to be a spherical space form (so that $\tilde{N} \cong S^{n}$ ), we can describe the homotopy groups of the wedge $\bigvee^{k} S^{n} \vee \tilde{N}$ as a direct sum of the homotopy groups of spheres $S^{j(n-1)+1}$ (cf. $\left.[\mathrm{H}]\right)$; often this allows explicit calculations.

QUESTION A. What can be said about the homotopy groups of the wedge $\bigvee^{k} S^{n} \vee \tilde{N}$ for general manifolds?

We have the logical implications

$$
\left(f_{1}, f_{2}\right) \text { is loose } \Rightarrow \omega^{\#}\left(f_{1}, f_{2}\right)=0 \Leftrightarrow \mathrm{N}^{\#}\left(f_{1}, f_{2}\right)=0
$$

(cf. [K4], theorem 1.30). Of course it is of central importance to know when $\omega^{\#}$ and $\mathrm{N}^{\#}$ are complete looseness obstructions, i.e. when the vanishing of $\omega^{\#}\left(f_{1}, f_{2}\right)$ or, equivalently, 
of $\mathrm{N}^{\#}\left(f_{1}, f_{2}\right)$ is also sufficient for the pair $\left(f_{1}, f_{2}\right)$ to be loose. Since

$$
\omega^{\#}\left(\left[f_{1}\right],\left[f_{2}\right]\right)=\omega^{\#}\left(\left[f_{1}\right]-\left[f_{2}\right], 0\right)+\omega^{\#}\left(\left[f_{2}\right],\left[f_{2}\right]\right)
$$

the following two settings are of particular interest.

I. The root case: $f_{2} \equiv y_{0}$ (where the fixed value $y_{0} \in N$ is given). Here our invariant yields the degree homomorphism

$$
\operatorname{deg}^{\#}:=\omega^{\#}\left(-, y_{0}\right): \pi_{m}(N) \rightarrow \pi_{m}\left(S^{n} \wedge(\Omega N)^{+}\right)
$$

which turns out to be essentially an enriched Hopf-Ganea-invariant homomorphism (see [K2], theorem 7.2). It is also related to the homomorphism

$$
\left(\operatorname{pinch}_{*}, \partial\right): \pi_{m}\left(\tilde{N}, \tilde{N} \backslash \bigcup^{k} \stackrel{\circ}{B^{n}}\right) \rightarrow \pi_{m}\left(\bigvee^{k} S^{n} \vee \tilde{N}, \tilde{N}\right) \oplus \pi_{m-1}\left(\tilde{N} \backslash \bigcup^{k} \stackrel{\circ}{B^{n}}\right)
$$

(cf. [K2], 7.3) where $\bigcup^{k} \stackrel{\circ}{B^{n}}$ is the disjoint union of open $n$-balls whose (embedded) boundary spheres intersect only in $\tilde{y}_{0} \in \tilde{N}$; the "pinching map" pinch collapses these boundary spheres into a single point and deforms $\tilde{N} \backslash \cup B^{n}$ to $\tilde{N} \backslash\left\{\tilde{y}_{0}\right\} ; \partial$ denotes the obvious connecting homomorphism.

Clearly deg ${ }^{\#}$ vanishes on $i_{*}\left(\pi_{m}\left(N \backslash\left\{y_{0}\right\}\right)\right)$ where $i$ denotes the inclusion $N \backslash\left\{y_{0}\right\} \subset N$. The following conditions are equivalent (cf. [K2], 6.4 and 7.3):

(i) the sequence

$$
\pi_{m}\left(N \backslash\left\{y_{0}\right\}\right) \stackrel{i_{*}}{\longrightarrow} \pi_{m}(N) \stackrel{\mathrm{deg}^{\#}}{\longrightarrow} \pi_{m}\left(S^{n} \wedge(\Omega N)^{+}\right)
$$

is exact;

(ii) the homomorphism (pinch, $\partial$ ) (cf. (8)) is injective;

(iii) $\operatorname{deg}^{\#}(f)$ is the complete looseness obstruction for all pairs of the form $\left(f, y_{0}\right)$, where $f: S^{m} \rightarrow N$.

All these conditions are often satisfied, e.g. when $m \leq 2 n-3$ or $n=2$ or $N$ is a sphere or a (real, complex or quaternionic) projective space of arbitrary dimension (cf. [K2], theorem 6.5). Do they always hold?

QUESTION B. What can be said about the kernel of (pinch, $\partial)(c f .(8))$ for general $N$ and arbitrary dimensions?

It may also be interesting to note that the Nielsen number $\mathrm{N}^{\#}\left(f, y_{0}\right)$ can assume only the values 0 and $k$ (cf. [K2], 4.3).

II. The selfcoincidence case: $f_{1}=f_{2}=: f$. Here the loopspace aspect of our invariants carries no extra information. Therefore $\omega^{\#}(f, f)$ is precisely as strong as its image in $\pi_{m}\left(S^{n}\right)$ under the obvious forgetful map, and the Nielsen Number $\mathrm{N}^{\#}(f, f)$ can take only the values 0 and 1 . However, we can distinguish between two kinds of deformations: small deformations (which move $f$ only a distance $\varepsilon$ away for a small $\varepsilon>0$ ) on one hand, and arbitrary deformations (which may use all the space available in $N$ ) on the other hand (cf. $[\mathrm{DG}])$. 
Consider the bundle $S T(N)$ of unit tangent vectors over $N$ (with respect to some metric) and the resulting exact (horizontal) homotopy sequence as well as the Freudenthal suspension homomorphism $E$ :

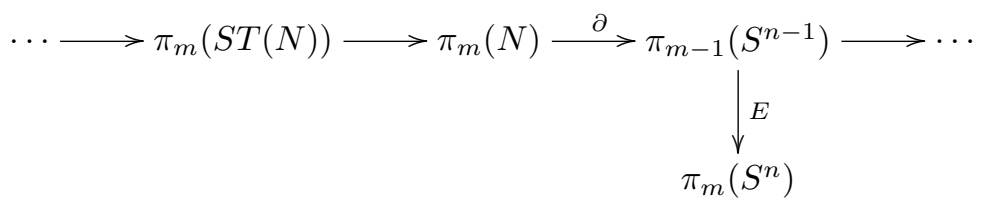

Theorem 3. Given $[f] \in \pi_{m}(N)$, we have the following logical implications:

(i) $\partial([f]) \in \pi_{m-1}\left(S^{n-1}\right)$ vanishes;

立

(ii) $(f, f)$ is loose by small deformation;

$\Downarrow \quad(\Uparrow$ if $N=\mathbb{R P}(n))$

(iii) $(f, f)$ is loose (by any deformation);

$\Downarrow \quad\left(\Uparrow \quad\right.$ if $\left.N=S^{n}\right)$

(iv) $\omega^{\#}(f, f)=0$;

$\Uparrow$

(v) $E(\partial([f])=0$.

Thus $\omega^{\#}(f, f)$ is just "one desuspension short" of being the complete looseness obstruction.

The equivalence of (i) and (ii) was already noted by A. Dold and D. L. Gonçalves in $[\mathrm{DG}]$.

Observe also that all the conditions (i), ., (v) above except (iii) are compatible with covering projections $p: \tilde{N} \rightarrow N$ (compare [K3], 1.22).

COROLlary 4. The conditions (i),..,(v) in theorem 3 are all equivalent if the suspension homomorphism $E$, when restricted to $\partial\left(\pi_{m}(N)\right)$ (cf. (9)), is injective and, in particular, if $m \leq n+3$ or if $m=n+4 \neq 10$ or in the stable dimensional range $m \leq 2 n-3$.

Indeed, in these three dimension settings $E$ is injective whenever $n \equiv 0(2)$ (compare e.g. [T] or [K4], 4.5). If the Euler characteristic of $N$ vanishes (e.g. when $n \not \equiv 0(2)$ ), then the conditions (i), ., (v) in theorem 3 are automatically satisfied due to the existence of a nowhere vanishing vector field along which the map $f$ can be pushed slightly away from itself.

Corollary 5. Assume that

(i) $\# \pi_{1}(N)>2$ and $N$ is orientable or not; or

(ii) $\# \pi_{1}(N) \geq 2$ and $N$ is orientable.

If in addition $\left.E\right|_{\partial\left(\pi_{m}(N)\right)}$ is injective, then for all maps $f: S^{m} \rightarrow N$ the pair $(f, f)$ is loose by small (and hence by any) deformation.

Indeed, according to theorem 1.21 of $[\mathrm{K} 4], \omega^{\#}(f, f)$ vanishes here.

This suggests that our invariants are particularly interesting when $N$ has a small fundamental group, e.g. in the case of spheres and projective spaces. 
TheOREM 6. Given a map $f: S^{m} \rightarrow \mathbb{R P}(n), m, n \geq 2$, let $\tilde{f}: S^{m} \rightarrow S^{n}$ be a lifting. Then the following conditions are equivalent:

(i) $\omega^{\#}(f, f)=0$, but $(f, f)$ is not loose;

(ii) $(\tilde{f}, \tilde{f})$ is loose but not by small deformation;

(iii) $\partial_{\mathbb{R}}([\tilde{f}]) \neq 0$, but $E \circ \partial_{\mathbb{R}}([\tilde{f}])=0$;

(iv) $(\tilde{f}, \tilde{f})$ is loose, but $(f, f)$ is not loose;

(v) $\operatorname{MC}(\tilde{f}, \tilde{f})<\operatorname{MC}(f, f)$;

(vi) $\operatorname{MCC}(\tilde{f}, \tilde{f})<\operatorname{MCC}(f, f)$.

In particular, maps $f, \tilde{f}$ which satisfy these conditions exist precisely in those dimension combinations where $E$ is not injective on $\partial\left(\pi_{m}\left(S^{n}\right)\right)$.

Already in the first nonstable dimension setting we encounter fascinating interrelations with other, seemingly distant, branches of topology.

THEOREM 7. Let $\tilde{f}: S^{2 n-2} \rightarrow S^{n}$ be a lifting of a map $f: S^{2 n-2} \rightarrow \mathbb{R P}(n)$. Assume that $n$ is even, $n \neq 2,4,8$. Then the pair $(f, f)$ is loose if and only if both $\omega^{\#}(f, f)$ and the Kervaire invariant $\mathrm{K}([\tilde{f}])$ of $\tilde{f}$ vanish.

Such a connection with the Kervaire invariant was originally discovered by D. L. Gonçalves and D. Randall [GR2] who also studied the second nonstable dimension setting (in [GR1]) and found the first examples illustrating a version of the following result (cf. $[\mathrm{KR}])$.

THEOREM 8. Let $\tilde{f}: S^{2 n-1} \rightarrow S^{n}$ be a lifting of a map $f: S^{2 n-1} \rightarrow \mathbb{R P}(n)$. Assume that $n \equiv 2(4), n \geq 6$. Then $(f, f)$ is loose if and only if $\omega^{\#}(f, f)=0$ and, in addition, the Hopf invariant $\mathrm{H}(\tilde{f})$ is divisible by 4 .

In the next nonstable dimension settings the noninjectivity of $\left.E\right|_{\partial\left(\pi_{m}\left(S^{n}\right)\right)}$ turns out to be closely related to the question whether the Whitehead products of $\iota_{n-1}$ with $\eta_{n-1}^{2}, \nu_{n-1}, \nu_{n-1}^{2}, \sigma_{n-1}, \ldots$ can be halved, i.e. lie in $2 \pi_{*}\left(S^{n-1}\right)$. Many relevant results have been listed by M. Golasiński and J. Mukai in their very helpful paper [GM], but what else is known?

Question C. Which Whitehead products in $\pi_{m-1}\left(S^{n-1}\right)$, $n$ even, are divisible by 2 ?

This seems to be a subtle problem; in coincidence theory it arises already in the very special case where $N$ equals $S^{n}$ or $\mathbb{R P}(n)$.

Question D. What can be said about the subgroup $\partial\left(\pi_{m}(N)\right)$ of $\pi_{m-1}\left(S^{n-1}\right)(c f .(9))$ for arbitrary manifolds $N$ with nontrivial Euler characteristic?

When is $\left.E\right|_{\partial\left(\pi_{m}(N)\right)}$ injective?

E.g. what about the case where $N$ is a general Grassmannian manifold?

\section{References}

[B] R. Brown, Wecken properties for manifolds in: Contemp. Math. 152, Amer. Math. Soc., 1993, 9-21. 
[DG] A. Dold and D. L. Gonçalves, Self-coincidence of fibre maps, Osaka J. Math. 42 (2005), 291-307.

[GM] M. Golasiński and J. Mukai, Gottlieb groups of spheres, Topology 47 (2008), 399-430.

[GR1] D. L. Gonçalves and D. Randall, Self-coincidence of maps from $S^{q}$-bundles over $S^{n}$ to $S^{n}$, Bol. Soc. Mat. Mexicana (3) 10 (2004), 181-192.

[GR2] D. L. Gonçalves and D. Randall, Self-coincidence of mappings between spheres and the strong Kervaire invariant one problem, Comptes Rendus Math. Acad. Sci. Paris Sér. I 342 (2006), 511-513.

$[\mathrm{H}] \quad$ P. Hilton, On the homotopy groups of the union of spheres, J. London Math. Soc. f30 (1955), 154-172.

[K1] U. Koschorke, Nielsen coincidence theory in arbitrary codimensions, J. Reine Angew. Math. 598 (2006), 211-236.

[K2] U. Koschorke, Nonstabilized Nielsen coincidence invariants and Hopf-Ganea homomorphisms, Geom. Top. 10 (2006), 619-665.

[K3] U. Koschorke, Minimizing coincidence numbers of maps into projective spaces, Memorial Volume in honor of H. Zieschang, Geom. Top., to appear. (The papers [K1]-[K3] can be found at http://www.math.uni-siegen.de/topology/publications.html)

[K4] U. Koschorke, Selfcoincidences and roots in Nielsen theory, J. Fixed Point Theory Appl. 2 (2007), 241-259.

[KR] U. Koschorke and D. Randall, Kervaire invariants and selfintersections, preprint, New Orleans, 2007.

[T] H. Toda, Composition Methods in Homotopy Groups of Spheres, Princeton Univ. Press, 1962. 